\title{
Complementary DNA sequences encoding the multimammate rat MHC class II DQ $\alpha$ and $\beta$ chains and cross-species sequence comparison in rodents
}

\author{
J. Goüy de Bellocq ${ }^{1} \&$ H. Leirs ${ }^{1,2}$ \\ 1 Department of Biology, University of Antwerp, Groenenborgerlaan 171, 2020 Antwerp, Belgium \\ 2 Danish Pest Infestation Laboratory, Department of Integrated Pest Management, University of Aarhus, Skovbrynet 14, DK-2800 Kongens \\ Lyngby, Denmark
}

\author{
Key words \\ DQ; major histocompatibility complex class II; \\ multimammate rat; Muridae; RACE \\ Correspondence \\ Joëlle Goüy de Bellocq \\ Department of Biology \\ University of Antwerp \\ Groenenborgerlaan 171 \\ 2020 Antwerp \\ Belgium \\ Tel: + 3232653469 \\ Fax: + 3232653474 \\ e-mail: joelle.gouydebellocq@ua.ac.be
}

Received 13 February 2009; revised 19 May

2009; accepted 3 June 2009

doi: 10.1111/j.1399-0039.2009.01305.x

\begin{abstract}
Sequences of the complete open reading frame (ORF) for rodents major histocompatibility complex (MHC) class II genes are rare. Multimammate rat (Mastomys natalensis) complementary DNA (cDNA) encoding the $\alpha$ and $\beta$ chains of MHC class II DQ gene was cloned from a rapid amplifications of cDNA Emds (RACE) cDNA library. The ORFs consist of 801 and 771 bp encoding 266 and 256 amino acid residues for DQB and DQA, respectively. The genomic structure of Mana- $D Q$ genes is globally analogous to that described for other rodents except for the insertion of a serine residue in the signal peptide of Mana- $D Q B$, which is unique among known rodents.
\end{abstract}

The Muridae is the largest family of mammals with $>730$ recognized species (1), including two classical laboratory models, the mouse (Mus musculus) and rat (Rattus norvegicus), and many reservoirs of human diseases [e.g. reservoirs of old world arenaviruses causing haemorrhagic fevers (2)]. To date, the genetic structure of the major histocompatibility complex (MHC) of Muridae has been intensively investigated in the two laboratory models - labelled as the H2 system in the mouse and the RT1 complex in the rat (3-5).

Mastomys natalensis, the multimammate rat, is a widely distributed African murid (6), a pest causing agricultural damage and/or acting as a natural reservoir of human pathogens (7). In West Africa, M. natalensis is the reservoir of Lassa virus, an arenavirus causing haemorrhagic fever (8). In southern Africa, M. natalensis plays a role in the transmission of the bacteria Yersinia pestis - the aethiologic agent of plague - between wild and commensal rodents
(9). M. natalensis is used as laboratory model of human diseases such as papillomavirus infections (10) and for the investigation of bilharziasis, filariasis, relapsing fever and plague $(11,12)$. It is surprising, then, that the multimammate rat MHC has never been characterized.

The primary aim of our work was to clone transcripts of M. natalensis MHC class II DQ and thereby provide data for further studies on pathogen resistance in natural populations. As sequences encoding the complete open reading frame (ORF) of DQ genes are available for a few other rodent species, we were able to compare the complementary DNA (cDNA) sequences of the multimammate rat with those of other rodents and relate them to those of other mammal species.

The individuals were obtained from a laboratory colony established in 2006 in the Danish Pest Infestation Laboratory. Progenitors of the colony were captured in Morogoro, 
Tanzania, in 2006. Four individuals were randomly chosen from the F1 generation. Total RNA was isolated from liver preserved in RNAlater using RNeasy mini kit with oncolumn RNase-free DNase set (Qiagen Vertriebs $\mathrm{GmbH}$, Wien, Austria). RNA from the four individuals was pooled two by two into two samples and used as a template for full-length cDNA synthesis using an RNA ligase-mediated RACE with the GeneRacer kit (Invitrogen $\mathrm{GmbH}$, Lofer, Austria). Polymerase chain reaction (PCR) amplifications of both the $5^{\prime}$ and the $3^{\prime}$ ends of the cDNA were performed in two separate reactions using primers provided with the RACE kit and primers designed from mouse and rat sequences retrieved from GenBank: GeneRacer 5' Primer and MasDQA-Ex3R-Race or MusDQB-R2 for the DQA and DQB, respectively, for the amplification of the $5^{\prime}$ ends; MasDQA-Ex2F-Race or BetaUniv-F and GeneRacer 3' Primer for the DQA and DQB, respectively, for the amplification of the $3^{\prime}$ ends. PCRs were performed using a Multiplex PCR kit (Qiagen) in a final reaction volume of $35 \mu$ l. Primers provided with the RACE kit were used at the final concentration of $0.5 \mu \mathrm{M}$ and primers were designed for Mastomys at $0.2 \mu \mathrm{M}$. The thermal profile started with an initial HotStarTaq DNA Polymerase activation at $95^{\circ} \mathrm{C}$ (15 min), followed by 5 cycles at $94^{\circ} \mathrm{C}(30 \mathrm{~s})$ and $72^{\circ} \mathrm{C}(75 \mathrm{~s})$, followed by 5 cycles at $94^{\circ} \mathrm{C}(30 \mathrm{~s})$ and $70^{\circ} \mathrm{C}(75 \mathrm{~s})$, followed by 25 cycles at $94^{\circ} \mathrm{C}(30 \mathrm{~s}), 66.5^{\circ} \mathrm{C}(30 \mathrm{~s})$ and $72^{\circ} \mathrm{C}(75 \mathrm{~s})$ and ending with an extension step at $72^{\circ} \mathrm{C}(10 \mathrm{~min})$. The PCR products were electrophoresed on $1.5 \%$ agarose gels and visualized by ethidium bromide staining. Bands within the expected size range were purified using S.N.A.P. columns. The PCR products were cloned using TOPO TA cloning kit (Invitrogen). Plasmid DNA of positive transformants was extracted by QIAprep Spin Miniprep Kit (Qiagen) and sequenced (Macrogen, Seoul, Korea) in both directions using 5'M13 Reverse and 3'T7 promoter universal primers. For the DQA gene, we sequenced four plasmid inserts by sample for the $5^{\prime}$ end and five plasmid inserts for only one of the samples for the $3^{\prime}$ end. For the DQB gene, we sequenced six plasmid inserts by sample for both $5^{\prime}$ and $3^{\prime}$ ends. To assign the transcribed sequences to the mammal DQ gene, the cDNA sequences were compared with transcribed sequences from other Muridae and rodents. Nomenclature of the M. natalensis $D Q$ (Mana-DQ) allelic sequences follows Klein et al. (13). M. natalensis MHC class II DQ exon layout is relative to the exon structure of the rat, mouse and human DQ cDNAs described in Ettinger et al. (14), which is in turn based on a structure-based study (15). The cleavage site of the signal peptide was predicted using SignalP 3.0 (16).

For the DQB gene, the entire coding sequence and some $5^{\prime}$ and $3^{\prime}$ untranslated regions (UTR) were amplified by RACE. The 5' cDNA ends amplified using GeneRacer 5' and MusDQB-R2 primers were $\sim 400 \mathrm{bp}$ long. Three different sequences were obtained from the 12 sequenced plasmid inserts: one sequence was identified by BLAST as a DRB allele and the remaining sequences as two different DQB alleles. The $3^{\prime}$ cDNA ends amplified using the GeneRacer 3' and BetaUniv-F primers were $~ 900$ bp long. Four different sequences were obtained from the 12 sequenced plasmid inserts: 3 were identified as coding for the DQB gene and the last one for the haemopexin gene (BLAST 95\% identity with haemopexin of $M$. musculus). Comparing the 168 shared nucleotides between the $5^{\prime}$ and the 3' cDNA ends, two of the 3' end sequences matched the two $5^{\prime}$ end sequences. Sequences were named Mana-DQB*01 and Mana-DQ $B^{*} 02$ for the two complete coding sequences and Mana-DQB*03 for the incomplete $3^{\prime}$ end coding sequence (GenBank AN: FJ968784-FJ968786). The nucleotide sequences showed high similarity with rodent sequences (BLAST: identity with M. musculus: 90\%, R. norvegicus: $89 \%$ and Peromyscus eremicus: $87 \%$ ). The $\sim 1200$ bp contain an 801 bp ORF coding 266 residues (Figure 1).

For the DQA gene, the entire coding sequence with some 5' and 3' UTRs was also successfully amplified by RACE. The 5' cDNA ends amplified using GeneRacer 5' and MasDQA-Ex3R-Race primers were $\sim 400$ bp long. Three different sequences were obtained from the eight sequenced plasmid inserts. The sequences were identified by BLAST as encoding the DQA gene. The 3' cDNA ends amplified using the GeneRacer 3' and MasDQA-Ex2F-Race primers were $\sim 850$ bp long. Three different sequences were obtained from the five sequenced plasmid inserts and were identified as coding for DQA gene. Comparing the 168 shared nucleotides between the $5^{\prime}$ and the $3^{\prime}$ cDNA ends, one of the $3^{\prime}$ end sequence matched one of the $5^{\prime}$ end sequence. DQA sequences were named Mana-DQA*01 for the complete coding sequence, Mana-DQA*02 and Mana-DQA*03 for the incomplete $5^{\prime}$ end coding sequences and Mana-DQ $A^{*} 04$ and Mana-DQA*05 for the incomplete $3^{\prime}$ end coding sequences (AN: FJ968787-FJ968791). The nucleotide sequences showed high homology with rodent sequences (BLAST: identity with M. musculus: 91\%, R. norvegicus: 90\% and Sigmodon hispidus: $88 \%$ ). The $\sim 1100$ bp contain a 771 bp ORF coding 256 residues (Figure 2).

The deduced amino acid sequences were aligned and numbered according to the structure of RT1-B of the rat (14) and H2-IA of the mouse (17) (Figures 1 and 2). SIGNALP predicted the signal peptide cleavage site between Gly and Arg at -1 and 1 and between Gly and Gln/Glu at -1 and 1 a for the mature Mana-DQB and Mana-DQA, respectively. The predicted signal peptide cleavage sites were similar to those for other rodents and mammals, although the human, cattle and dog signal peptides of DQB are four amino acids longer than that of Mastomys (14). The $\beta 65-66$ deletion, very common in rat and mouse haplotypes $(14,17)$, was not found in the three Mastomys sequences (Figure 1). In rodents, this deletion has only been described in mouse and rat so far, appearing absent from the two other rodent genera, Peromyscus and Ctenomys $(18,19)$. The $\beta 84 \mathrm{a}$ 


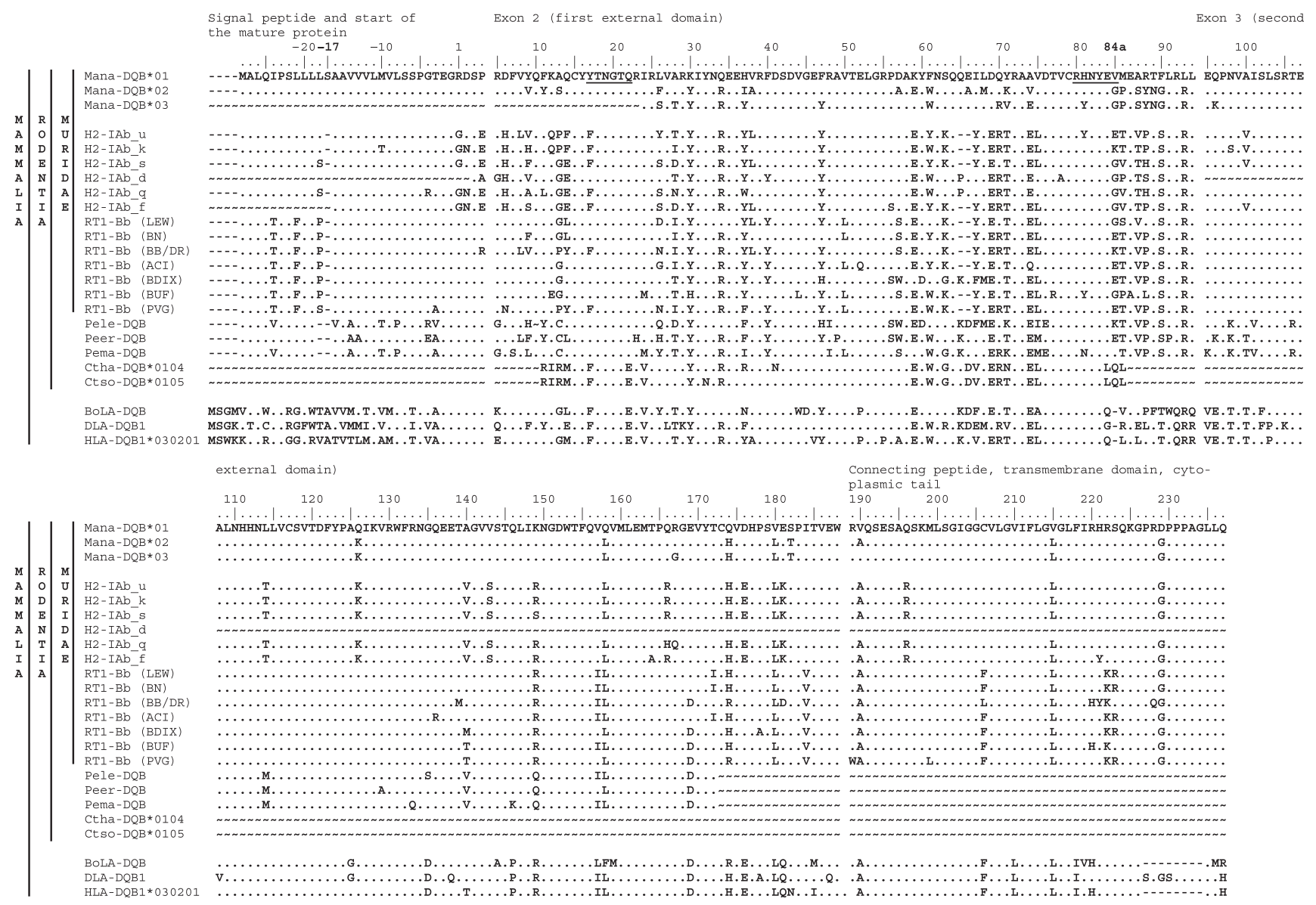

Figure 1 Comparison of the translated amino acid sequences of Mastomys natalensis major histocompatibility complex class II DQB gene (Mana-DQB) and other representative of murids, rodents and mammals. Sequences are split according to their domain structure. The symbols used indicate the following: -, indel; ., same amino acids as those in Mana-DQB*01; , alignment filler. Numbering of Mana-DQB includes two insertions of amino acids designated -17 and 84a (in bold). The sources of the sequences are as follows: Mus musculus H2-lab (GenBank accession number: M13537-M13541 and AH002012), Rattus norvegicus RT1-Bb (AY626180-AY626183, AY626186, AY626187 and AY626189), Ctenomys haigi Ctha-DOB (AF312534), Ctenomys sociabilis Ctso-DQB (AF312530), Peromyscus leucopus Pele-DQB (AF300846), Peromyscus eremicus Peer-DQB (AF300853), Peromyscus maniculatus Pema-DQB (AF300855), Bos taurus BoLA-DQB (Y18201), Canis lupus familiaris DLA-DQB (NM_001014381) and Homo sapiens sapiens HLA-DQB1 *030201 (IMGT/HLA AN: HLA00627). Positions of primers used for the RACE, BetaUniv-F (5'-acttcaccaacgggacgcagc-3') and MusDOB-R2 $\left(5^{\prime}\right.$-cccctcgtagttgtgtctgc-3') are underlined. HLA, human leucocyte antigen.

insertion described in mouse and rat $(16,17)$ was also found in the multimammate rat and seems to be a characteristic shared by all rodent species (Figure 1). The Mana-DQB amino acid sequences differ from those of other rodents because of insertion of a Ser residue in the signal peptide at position - 17. This residue is an Ala in $H L A-D Q B 1, D L A$ $D Q B 1$ and $B o L A-D Q B$ (Figure 1). The structural characteristics of Mana-DQA did not, in contrast, differ from those of other rodents (Figure 2).

The numbering of Mana- $D Q B$ and Mana-DQA was checked by identifying the position of evolutionary conserved amino acids: the Asn and Thr that provide a site for carbohydrate attachment were identified at amino acids $\beta 19$ and $\beta 21$; the Cys that form an intradomain disulfide bond was identified at amino acids $\beta 15$ and $\beta 79$; the Asn and
Arg that form a hydrogen bonds with the backbone of the peptide were identified at amino acids $\beta 82$ and $\alpha 76$, respectively $(15,20)$.

In summary, we have characterized cDNA encoding the entire mature proteins of the Mana-DQA and $D Q B$ class II molecules. Comparison with other rodent DQ proteins identifies a major discrepancy in the leader peptide of the Mana- $D Q B$ protein, which is insertion of a Ser residue. There has been no study investigating the functional role of polymorphism in the signal peptide in MHC class II molecules. However, this region shows some polymorphism in humans [e.g. DQB1 (21)], and in addition to the insertion of the Ser residue in the signal peptide of Mana-DQB compared with DQB sequences in other rodents, the signal peptide of the Mana-DQA shows one polymorphic site (Figure 2). In 


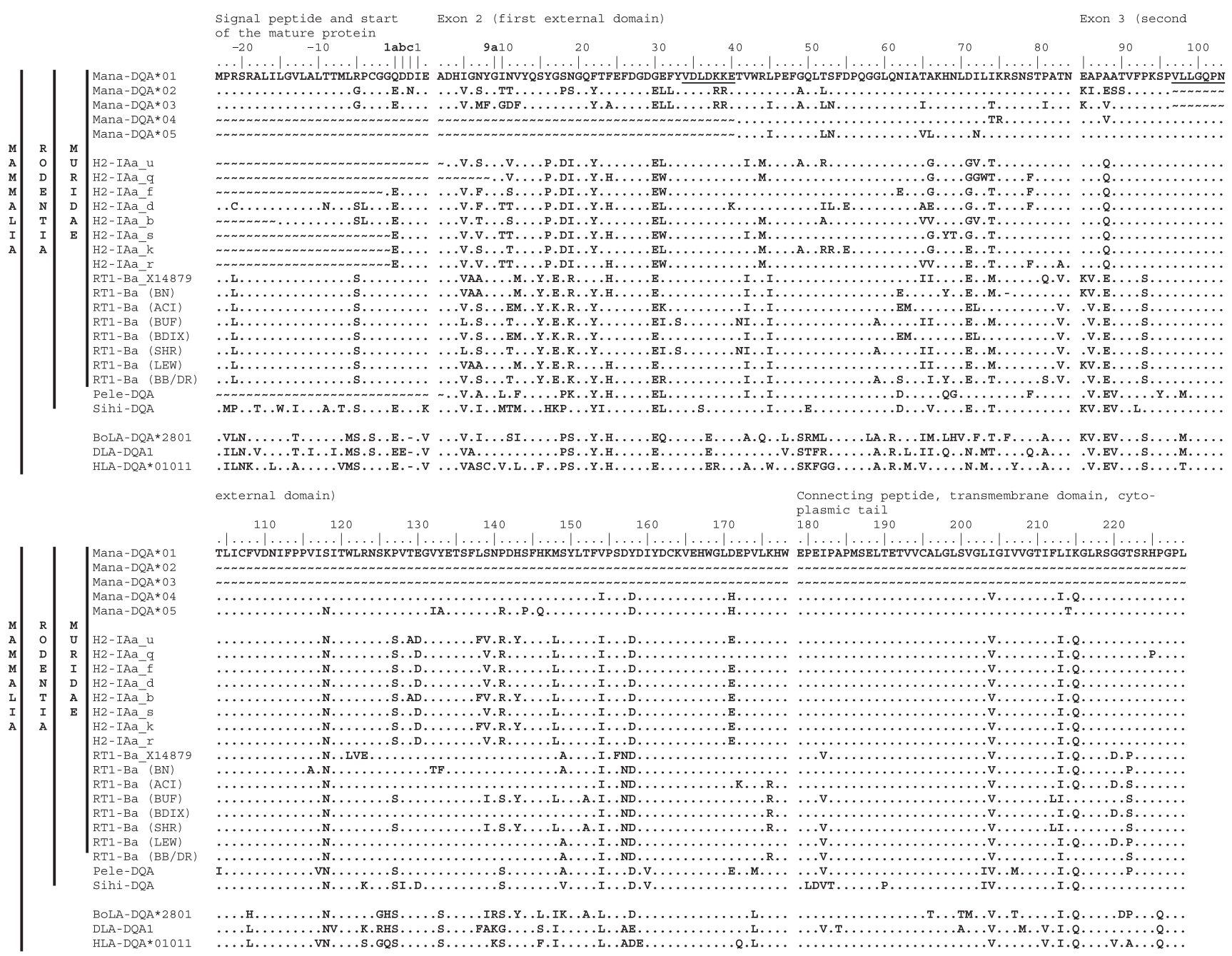

Figure 2 Comparison of the translated amino acid sequences of Mastomys natalensis MHC class II DQA gene (Mana-DQA) and other representative of murids, rodents and mammals. Sequences are split according to their domain structure. Numbering of Mana-DQA includes an insertion in exon 2, designated amino acid 9a and three extra amino acids at the start of the mature protein designated $1 \mathrm{a}, 1 \mathrm{~b}$ and $1 \mathrm{c}$ (in bold). These numbers are inserted for structural consistency across $\mathrm{MHC}$ class II molecules (15). The symbols used indicate the following: -, indel; ., same amino acids as those in Mana-DQA*01; , alignment filler. The sources of the sequences are as follows: Mus musculus H2-IAa (K01922-K01926 and M11356-M11358), Rattus norvegicus RT1-Ba (X14879, AY626190, AY626191, AY626193, AY626194, AY626196, AY626197 and AY626199), Peromyscus leucopus Pele-DQA (U34805), Sigmodon hispidus Sihi-DQA (AY169005), Bos taurus BoLA-DQA (AF037314), Canis lupus familiaris DLA-DQA1 (NM_001011726) and Homo sapiens sapiens HLA-DQA*01011(IMGT/HLA AN: HLA00601). Positions of the primers used for the RACE, MasDQA-Ex2F-Race (5'-atgtggacttggataagaaggag-3') and MasDQA-Ex3R-Race (5'-tgttgggctgacccagcagcac-3') are underlined. HLA, human leucocyte antigen; MHC, major histocompatibility complex.

humans, signal sequences of MHC class I molecules have been shown to play a role in NK-cell-mediated lysis through their presentation by the class I human leucocyte antigen-E molecule (22). The interspecific and intraspecific polymorphisms of signal peptide shown here suggest that the functional role of leader peptide sequences in MHC class II molecules deserves closer inspection.

\section{Acknowledgments}

The authors thank F. Suchentrunk and the Research Institute of Wildlife Ecology in Vienna for access to laboratory facilities. JGB is a postdoctoral fellow with the Fund for Scientific Research-Flanders (FWO). Financial support was provided by the University of Antwerp (grant number 2964) and the FWO (grant number 1.5.180.09).

\section{References}

1. Carleton MD, Musser GG. Order Rodentia. In: Wilson DE, Reeder DM, eds. Mammal Species of the World, 3rd edn. The Johns Hopkins University Press, Baltimore, 2005, 745-52.

2. Hugot JP, Gonzalez JP, Denys C. Evolution of the Old World Arenaviridae and their rodent hosts: generalized host-transfer or association by descent? Infect Genet Evol 2001: 1: 13-20. 
3. Gunther E, Walter L. The major histocompatibility complex of the rat (Rattus norvegicus). Immunogenetics 2001: 53: 520-42.

4. Hurt P, Walter L, Sudbrak R et al. The genomic sequence and comparative analysis of the rat major histocompatibility complex. Genome Res 2004: 14: 631-9.

5. Allcock RJ, Martin AM, Price P. The mouse as a model for effects of MHC genes on human disease. Immunol Today 2000: 21: $328-32$.

6. Granjon L, Duplantier J-M, Catalan J, Britton-Davidian J. Systematics of the genus Mastomys (Thomas, 1915) (Rodentia: Muridae). A review. Belg J Zool 1997: 127: 7-18.

7. Leirs H. Population Ecology of Mastomys natalensis (Smith, 1834). Implications for Rodent Control in Africa, Vol. 35. Brussels: Belgian Administration for Development Cooperation, Agricultural, ed., 1994.

8. Lecompte E, Fichet-Calvet E, Daffis S et al. Mastomys natalensis, Lassa fever West Africa. Emerg Infect Dis 2006: 12: 1971-4.

9. Davis DHS. Ecology of wild rodent plague. In: Davis DHS, De Meillon B, Harington JS, Kalk M, eds. Ecological Studies in Southern Africa. The Hague: Dr. W. Junk Publishers, 1964: 301-14.

10. Amtmann E, Volm M, Wayss K. Tumour induction in the rodent Mastomys natalensis by activation of endogenous papilloma virus genomes. Nature 1984: 308: 291-2.

11. Davis DHS. Wild rodents as laboratory animals and their contribution to medical research in South Africa. S Afr J Med Sci 1963: 28: 53-69.

12. Petranyi G, Mieth H, Leitner I. Mastomys natalensis as an experimental host for Brugia malaye subperiodic. Southeast Asian J Trop Med Public Health 1975: 6: 328-37.
13. Klein J, Bontrop RE, Dawkins RL et al. Nomenclature for the major histocompatibility complexes of different species: a proposal. Immunogenetics 1990: 31: 217-9.

14. Ettinger RA, Moustakas AK, Lobaton SD. Open reading frame sequencing and structure-based alignment of polypeptides encoded by $R T 1-B b, R T 1-B a, R T 1-D b$, and $R T 1-D a$ alleles. Immunogenetics 2004: 56: 585-96.

15. Kaufman J, Salomonsen J, Flajnik M. Evolutionary conservation of MHC class I and class II molecules - different yet the same. Semin Immunol 1994: 6: 411-24.

16. Dyrløv Bendtsen J, Nielsen H, von Heijne G, Brunak S. Improved prediction of signal peptides: SignalP 3.0. J Mol Biol 2004: 340: 783-95.

17. Fremont DH, Monnaie D, Nelson CA, Hendrickson WA, Unanue ER. Crystal structure of I-A ${ }^{\mathrm{k}}$ in complex with a dominant epitope of lysozyme. Immunity 1998: 8: 305-17.

18. Richman AD, Herrera LG, Nash D. Characterization of Peromyscus MHC class II beta sequences by ligation-anchored RT-PCR and denaturing gradient gel electrophoresis. Eur J Immunogenet 2002: 29: 213-7.

19. Hambuch TM, Lacey EA. Enhanced selection for MHC diversity in social tuco-tucos. Evolution 2002: 56: 841-5.

20. Reche PA, Reinherz EL. Sequence variability analysis of human class I and class II MHC molecules: functional and structural correlates of amino acid polymorphisms. J Mol Biol 2003: 331: 623-41.

21. Pascual M, Lopez-Nevot MA, Caballero A, Alonso A, Zanelli E, Martin J. Complete characterization of the DQB1 first exon polymorphism. Eur J Immunogenet 2002: 29: 447-8.

22. Lemberg MK, Bland FA, Weihofen A, Braud VM, Martoglio B. Intramembrane proteolysis of signal peptides: an essential step in the generation of HLA-E epitopes. J Immunol 2001:

167: 6441-6. 